\title{
Inadequação de macro e micronutrientes oferecidos em duas escolas de tempo integral públicas no Nordeste do Brasil
}

\author{
Inadequacy of macro and micronutrients on offer in two full-time \\ public schools in Northeastern Brazil
}

Neide Sheyla de Melo Araújo (https://orcid.org/0000-0001-9787-4967) ${ }^{1}$

Maria de Fátima Rebouças Antunes (https://orcid.org/0000-0003-3216-0255) ${ }^{2}$

Karla Maria Carneiro Rolim (http://orcid.org/0000-0002-7914-6939) ${ }^{1}$

Sara Maria Moreira Lima Verde (https://orcid.org/0000-0002-7733-0214) ${ }^{3}$

Shirley Christina Melo Araújo (https://orcid.org/0000-0001-9705-7473) ${ }^{4}$

Carlos Antônio Bruno da Silva (https://orcid.org/0000-0001-7478-2525) ${ }^{2}$

${ }^{1}$ Programa de PósGraduação em Saúde Coletiva, Universidade de Fortaleza. Av. Washington Soares 1321, Edson Queiroz. 60811-905 Fortaleza CE Brasil.neidesmaraujo@ hotmail.com

${ }^{2}$ Diretoria do Centro de Ciências da Saúde, Universidade de Fortaleza. Fortaleza CE Brasil. ${ }^{3}$ Centro de Ciências da Saúde, Universidade Estadual do Ceará (UECE). Fortaleza Ceará, Brasil.

${ }^{4}$ Faculdade de Saúde

Pública, Universidade de

São Paulo. São Paulo SP

Brasil.

\begin{abstract}
The National School Food Program guarantees the food of students in public and philanthropic schools by means of the transfer of funds. During adolescence, which is an important period of nutritional vulnerability, a healthy diet is responsible for the rates of physical growth and changes in body composition. The objective was to analyze the adequacy of macroand micronutrients provided to adolescents in full-time public schools in northeastern Brazil. The menus were evaluated using the parameters to meet $70 \%$ of the daily nutritional needs in terms of macronutrients and micronutrients of the students involved. It was found that, for most age groups, energy requirements were not met, attaining the maximum adequacy of $86.2 \%$, and protein ingestion was only sufficient on four days. The average amount of carbohydrates and lipids was below the recommended dose for all age groups. The levels of Vitamin $C$, zinc and calcium were insufficient in most of the meals on offer, though the iron quota was adequate for all age groups. The conclusion drawn is that enhanced planning needs to be incorporated in the preparation of menus to attempt to attain the requirements stipulated in the Program.

Key words School food, Public health, Adolescents, Nutrition and food programs and policies.
\end{abstract}

Resumo O Programa Nacional de Alimentação Escolar garante por meio da transferência de recursos financeiros a alimentação de alunos em escolas públicas e filantrópicas. Na adolescência, a boa nutrição é influenciada pelas taxas de crescimento fisico e de alterações na composição corporal, sendo um período importante de vulnerabilidade nutricional. Objetivou-se analisar a adequação de macro e micronutrientes oferecidos aos adolescentes de escolas públicas de tempo integral no Nordeste do Brasil. Os cardápios foram avaliados utilizando os parâmetros para o atendimento de $70 \%$ das necessidades nutricionais diárias de macronutrientes e micronutrientes dos alunos beneficiários. Nota-se que, na maioria das faixas etárias, não houve o atendimento das necessidades energéticas, atingindo o percentual máximo de adequação de 86,2\%. Houve adequação de proteinas em quatro dias apenas. O valor médio de carboidratos e lipídios estiveram abaixo do recomendado para todos os grupos etários. Os teores de Vitamina C, zinco e cálcio estiveram abaixo na maioria das preparações oferecidas. A quantidade de ferro foi alcançada em todas as faixas etárias. Conclui-se que deve haver um melhor planejamento na elaboração dos cardápios para tentar alcançar as necessidades previstas pelo Programa. Palavras-chave Alimentação escolar, Saúde pública, Adolescentes, Programas e politicas de nutrição e alimentação 


\section{Introdução}

O Programa Nacional de Alimentação Escolar (PNAE) implantado em 1955, garante por meio da transferência de recursos financeiros, a alimentação escolar de alunos de toda a educação básica (Educação Infantil, Ensino Fundamental, Ensino Médio e Educação de Jovens e Adultos) matriculados em escolas públicas e filantrópicas e busca atender as necessidades nutricionais destes alunos durante sua permanência em sala de aula, contribuindo para o crescimento, o desenvolvimento, a aprendizagem e o rendimento escolar dos estudantes, bem como promover a formação de hábitos alimentares saudáveis ${ }^{1}$.

Programas de alimentação escolar são destaques em diversos países como parte de sistemas de proteção social e ao possibilitar o desenvolvimento local sustentável, com a utilização de alimentos da produção agrícola local, cooperando com comunidades vulneráveis, atuando dentro da perspectiva de promover a Segurança Alimentar e Nutricional (SAN), em todas as suas dimensões, ou seja, disponibilidade, acesso e utilização dos alimentos e nutrientes ${ }^{2}$.

O fornecimento da alimentação para escolares é essencial para um adequado desenvolvimento das funções físicas e cognitivas ${ }^{1}$. A adolescência caracteriza-se por um período de grande e diversificada demanda nutricional que, devido à influências grupais e à percepção corpórea, pode resultar em hábitos alimentares inadequados e com repercussões importantes para a saúde ${ }^{3}$. As necessidades nutricionais neste período, são influenciadas pelas taxas de crescimento físico e de alterações na composição corporal, sendo, portanto, um período importante de vulnerabilidade nutricional, onde a maioria destes não alcançam as recomendações dietéticas, podendo induzir efeitos adversos ${ }^{4}$.

A Educação Integral constitui ação estratégica para garantir atenção e desenvolvimento integral às crianças, adolescentes e jovens, propiciando condições de atenção integral. Para tal, necessita ser conjugada com a proteção social, o que pressupõe políticas integradas que considerem, além da educação, outras demandas dos sujeitos, articuladas entre os campos da educação, do desenvolvimento social, da saúde, do esporte, da inclusão digital e da cultura. E, entre essas demandas está a alimentação desses alunos ${ }^{5}$.

Um dos objetivos do PNAE para escolas de Tempo Integral é a elaboração de cardápios que supram $70 \%$ das necessidades nutricionais diárias, distribuídas em, no mínimo, três refeições, assim atendendo às necessidades dos alunos com a maior jornada diária de aula ${ }^{6}$. Com essa ampliação, percebe-se a necessidade de reavaliação da alimentação oferecida, com vistas a suprir as necessidades dos alunos durante o maior período em que estes permanecem nas unidades de ensino, impondo consequentes alterações no planejamento e execução das refeições para as escolas integrantes do Programa ${ }^{7}$.

Os macronutrientes são macromoléculas nas estruturas vegetais e animais que podem ser digeridas, absorvidas e utilizadas por um outro organismo como fontes de energia. São divididos em três classes - carboidratos, gorduras e proteínas, necessárias para manter a integridade celular e do organismo. Fornecem energia e desempenham um papel protetor na saúde humana e processos bioquímicos no organismo. Já os micronutrientes têm relevante participação, na manutenção da homeostase do organismo, proliferação e diferenciação celular, função imune, proteção contra o estresse oxidativo, além de exercer papel importante na participação do metabolismo de muitos outros nutrientes ${ }^{8}$. A deficiência destes pode comprometer tais funções e desencadear ou exacerbar distúrbios metabólicos 9 .

O ferro é um micronutriente essencial para a maioria dos organismos vivos. Participa de múltiplos processos vitais variando desde mecanismos celulares oxidativo até transporte de oxigênio nos tecidos ${ }^{10}$. Uma dieta típica europeia fornece cerca de 15 miligrama de ferro e apenas $10 \%$ é absorvido ${ }^{11}$.

$\mathrm{Na}$ adolescência, a deficiência de ferro é uma condição complexa, na medida em que vários fatores podem estar envolvidos, uma vez que esta etapa da vida é marcada por intensas mudanças fisiológicas e psicológicas, interferências socioculturais (imagem corporal, influência dos pais e da mídia), além da possibilidade de condições econômicas desfavoráveis, o que pode ocasionar a anemia ferropriva nestes ${ }^{12}$.

Já a vitamina C (ácido ascórbico) está envolvida na síntese do colágeno e nos mecanismos de defesa antioxidante. Ressalta-se sua importância nutricional na adolescência, também, em relação ao aumento da biodisponibilidade do ferro, uma vez que estimula e aumenta sua absorção, e cujo requerimento está aumentado nesse ciclo da vida $^{13}$. O zinco é o segundo micronutriente com maiores concentrações no organismo humano e participa de muitas reações do metabolismo celular, incluindo processos fisiológicos, tais como função imune, defesa antioxidante, crescimento e desenvolvimento ${ }^{14}$. Uma dieta normal e equi- 
librada pode oferecer entre dez e 15 miligramas por dia de zinco. Dentre os alimentos mais ricos nesse micronutriente destacam-se peixes, carnes, aves, leite e derivados que podem oferecer cerca de $80 \%$ desse total ${ }^{3}$.

Pesquisas brasileiras têm mostrado que o padrão alimentar dos adolescentes é caracterizado pelo baixo consumo de alimentos como leite e produtos lácteos, frutas e hortaliças e pela alta ingestão de alimentos ricos em energia, gorduras saturadas, açúcares e sódio, tais como as bebidas açucaradas, guloseimas e biscoitos. Esse padrão de dieta pode estar prejudicando o consumo de alimentos ricos em cálcio. Crianças e adolescentes entre nove e 18 anos apresentam recomendação nutricional mínima de 1.100 miligramas de cálcio por dia, segundo a Estimated Average Requirements (EAR), não devendo ultrapassar o nível de ingestão máximo tolerável de 3.000 miligramas por dia. Leite e derivados, vegetais verdes escuros, certos tipos de peixes e oleaginosas representam importantes fontes alimentares do mineral ${ }^{15}$.

A Vitamina A é essencial ao crescimento e desenvolvimento do ser humano. Atua também na manutenção da visão, no funcionamento adequado do sistema imunológico e mucosas, que atuam como barreiras de proteção contra infecções. Apresenta especial importância durante os períodos de proliferação e de rápida diferenciação celular, tais como a adolescência ${ }^{13}$. A necessidade de vitamina A foi determinada pela Food and Nutrition Board/Institute of Medicine (2001), com base na ingestão necessária para assegurar uma reserva corpórea adequada. A partir da necessidade média estimada (EAR), de 625 microgramas por dia para homens e 500 microgramas por dia para mulheres, definiu-se a ingestão dietética recomendada (RDA) de 900 microgramas por dia para homens e 700 microgramas por dia para mulheres ${ }^{10}$.

A vitamina A é encontrada em alimentos de origem animal como vísceras (principalmente fígado), gemas de ovos e leite integral e seus derivados (manteiga e queijo). Os vegetais são fontes de vitamina A sob a forma de carotenóides (precursores de vitamina) os quais, no organismo, se converterão em vitamina A. Em geral, frutas e legumes amarelos e alaranjados e vegetais verde -escuros são ricos em carotenóides ${ }^{16}$.

A adolescência é um período de risco para adoção de práticas alimentares que propiciam consumo de alimentos com alta densidade energética e insuficiência de determinados nutrientes na dieta, entre os quais a fibra alimentar ${ }^{17}$. As evidências acumuladas indicam importante papel das deficiências de micronutrientes como fator predisponente, agravante na fisiopatologia de diversas doenças crônicas não transmissíveis, como as doenças cardiovasculares, a hipertensão, o diabetes mellitus, a obesidade, alguns tipos de câncer, a osteoporose, entre outras enfermidades ${ }^{13}$.

O desequilíbrio na ingestão de micronutrientes na fase da adolescência, torna essa fase ainda mais vulnerável, influenciando de forma desfavorável o desenvolvimento pubertário e o crescimento somático, o que justifica levantamentos e intervenções nutricionais nesse estágio de vida ${ }^{18}$. Em face disso, objetivou-se com o estudo avaliar a adequação de macro e micronurientes oferecidos aos adolescentes de escolas públicas de Tempo Integral no Nordeste do Brasil.

\section{Métodos}

Trata-se de um estudo de abordagem quantitativa de base documental realizada a partir da análise de fontes primárias constituída do mapa de cardápio oferecido em escolas estaduais. Estudo desenvolvido em duas escolas públicas (A e B) de ensino integral em Picos, estado do Piauí, onde a escola A atende adolescentes do Ensino Fundamental ( $6^{\circ}$ ao $9^{\circ}$ ano) e do Ensino Médio ( $1^{\circ}$ ao $3^{\circ}$ ano), compreendendo a faixa etária entre $12 \mathrm{e}$ 19 anos. A Escola B atende apenas a modalidade ensino médio (14 a 19 anos). Para análise da adequação de macro e micronutrientes foram utilizados cardápios da alimentação oferecida aos estudantes nos meses de outubro de novembro de 2019, disponibilizados pela Secretaria de Educação e Cultura do Estado do Piauí (SEDUC/PI).

Os cardápios oferecidos foram executados semanalmente e distribuídos em dois blocos de 15 dias. Ou seja, as preparações se repetiam na primeira e na terceira semana e na segunda e quarta semana (Quadro 1).

Foram avaliados dez dias de preparações do mapa de cardápio fornecido pelas nutricionistas da SEDUC/PI, sendo estas separadas em dias, de D1 a D10. O cardápio apresentado continha 30 refeições, organizadas de formas diferentes a serem duas preparações de lanches (manhã e tarde) e um almoço entre os intervalos dos lanches. Foram oferecidas três refeições distintas por dia (um lanche da manhã, um almoço e um lanche da tarde) por aluno, e se repetem a cada 15 dias. O mesmo cardápio era oferecido nas duas escolas de tempo integral.

Para a determinação das quantidades oferecidas dos nutrientes a serem estudados, levou- 
Quadro 1. Mapa de refeições oferecidas nas escolas de Tempo Integral A e B, 2019.

\begin{tabular}{|c|c|c|c|c|c|c|}
\hline \multirow{3}{*}{$\begin{array}{l}\text { Semanas } \\
1^{\mathrm{a}} \text { e } 3^{\mathrm{a}} \\
\text { semana }\end{array}$} & \multicolumn{6}{|c|}{ Refeição } \\
\hline & Refeição & $2^{\mathrm{a}}$ feira & $3^{a}$ feira & $4^{\mathrm{a}}$ feira & $5^{\mathrm{a}}$ feira & $6^{\mathrm{a}}$ feira \\
\hline & Lanche & $\begin{array}{l}\text { Pão com } \\
\text { margarina e } \\
\text { achocolatado }\end{array}$ & $\begin{array}{l}\text { Cuscuz com } \\
\text { ovo }\end{array}$ & $\begin{array}{l}\text { Mingau de } \\
\text { milho }\end{array}$ & $\begin{array}{l}\text { Rosca, } \\
\text { achocolatado }\end{array}$ & $\begin{array}{l}\text { Cuscuz com } \\
\text { linguiça }\end{array}$ \\
\hline & Almoço & $\begin{array}{l}\text { Baião, farofa de } \\
\text { calabresa, creme } \\
\text { de repolho. } \\
\text { Sobremesa } \\
\text { melancia }\end{array}$ & $\begin{array}{l}\text { Feijoada, } \\
\text { arroz branco, } \\
\text { salada. } \\
\text { Sobremesa } \\
\text { laranja }\end{array}$ & $\begin{array}{l}\text { Carne bovina } \\
\text { acebolada, } \\
\text { arroz branco, } \\
\text { salada. } \\
\text { Sobremesa } \\
\text { doce }\end{array}$ & $\begin{array}{l}\text { Frango assado, } \\
\text { arroz branco, } \\
\text { feijão e salada. } \\
\text { Sobremesa } \\
\text { banana }\end{array}$ & $\begin{array}{l}\text { Estrogonofe de } \\
\text { frango, arroz } \\
\text { branco, macarrão, } \\
\text { salada. Sobremesa } \\
\text { doce }\end{array}$ \\
\hline & Lanche & Suco com biscoito & $\begin{array}{l}\text { Cachorro- } \\
\text { quente }\end{array}$ & $\begin{array}{l}\text { Cuscuz com } \\
\text { salsicha }\end{array}$ & Iogurte & $\begin{array}{l}\text { Farinha láctea } \\
\text { com biscoitos }\end{array}$ \\
\hline \multirow[t]{3}{*}{$\begin{array}{l}2^{\mathrm{a}} \text { e } 4^{\mathrm{a}} \\
\text { semana }\end{array}$} & Lanche & Mingau de milho & $\begin{array}{l}\text { Rosca, } \\
\text { achocolatado }\end{array}$ & $\begin{array}{l}\text { Cuscuz com } \\
\text { linguiça }\end{array}$ & $\begin{array}{l}\text { Pão com } \\
\text { margarina e } \\
\text { achocolatado }\end{array}$ & Cuscuz com ovo \\
\hline & Almoço & $\begin{array}{l}\text { Estrogonofe de } \\
\text { frango, arroz } \\
\text { branco, macarrão, } \\
\text { salada. Sobremesa } \\
\text { doce }\end{array}$ & $\begin{array}{l}\text { Carne bovina } \\
\text { acebolada, } \\
\text { arroz branco, } \\
\text { salada. } \\
\text { Sobremesa } \\
\text { doce }\end{array}$ & $\begin{array}{l}\text { Frango assado, } \\
\text { arroz branco, } \\
\text { feijão e salada. } \\
\text { Sobremesa } \\
\text { banana }\end{array}$ & $\begin{array}{l}\text { Feijoada, } \\
\text { arroz branco, } \\
\text { salada. } \\
\text { Sobremesa } \\
\text { laranja }\end{array}$ & $\begin{array}{l}\text { Baião, farofa de } \\
\text { calabresa, creme } \\
\text { de repolho. } \\
\text { Sobremesa } \\
\text { melancia }\end{array}$ \\
\hline & Lanche & Suco com biscoito & Iogurte & $\begin{array}{l}\text { Farinha láctea } \\
\text { com biscoitos }\end{array}$ & $\begin{array}{l}\text { Cuscuz com } \\
\text { salsicha }\end{array}$ & Cachorro-quente \\
\hline
\end{tabular}

Fonte: SEDUC/PI, 2019.

se em consideração o valor per capita de cada refeição oferecidos aos alunos. Esta informação foi fornecida como parte do cardápio, segundo os ingredientes utilizados nas preparações e estimados em programa de avaliação de composição química de alimentos.

Os per capitas utilizados foram os preconizados pelo Programa, com algumas adaptações em relação ao fechamento dos valores fornecidos pelo governo federal (RS 0,90), juntamente com o governo estadual $(\mathrm{R} \$ 1,10)$ totalizando o valor de $\mathrm{R}$ 2,00 por refeição/dia/aluno. O cardápio foi separado em ingredientes e a partir da composição e do per capita, foram avaliados o teores de energia (Kcal), através das estimativas de Requerimentos Energéticos (do inglês, EER); dos macronutrientes carboidratos, lipídios e proteínas (AMDR), e de fibras e de micronutrientes ferro, vitamina $\mathrm{C}$ (ácido ascórbico), zinco, cálcio, vitamina A e fibras pela DRI ${ }^{19}$.

Os cardápios foram avaliados de acordo com as recomendações do PNAE, utilizando os parâmetros para o atendimento de $70 \%$ das necessidades nutricionais diárias de macronutrientes e micronutrientes dos alunos beneficiários, considerando que estes realizam três refeições na escola.
A partir da quantidade e da composição nutricional de cada refeição, calculou-se a quantidade de nutrientes oferecidos diariamente, a média semanal, bem como a sua adequação segundo as referências por grupo de idade estudados (11-15 anos/16-18 anos/19-30 anos) preconizadas pelo PNAE.

\section{Resultados}

Foram analisadas as escolas A e B que atendem, respectivamente, 132 e 261 alunos, num total de 393. Os alunos foram distribuídos de acordo com o gênero, onde na escola A a maioria era do sexo feminino - 70 alunos (53\%) e 62 alunos do sexo masculino (47\%). Já na escola B, a maioria era do sexo masculino correspondendo a $54 \%$ do total $(n=141)$ e cerca de $46 \%$ do sexo feminino $(n=120)$.

Dos alunos citados na escola A, a maior frequência ocorreu na idade de 16 anos num total de $49(56,3 \%)$ alunos, com 25 mulheres e 24 homens. A escola B atende cerca de 261 alunos, maioria encontra-se na faixa etária de 14 anos, num total de $66(25 \%)$ alunos. No total as duas 


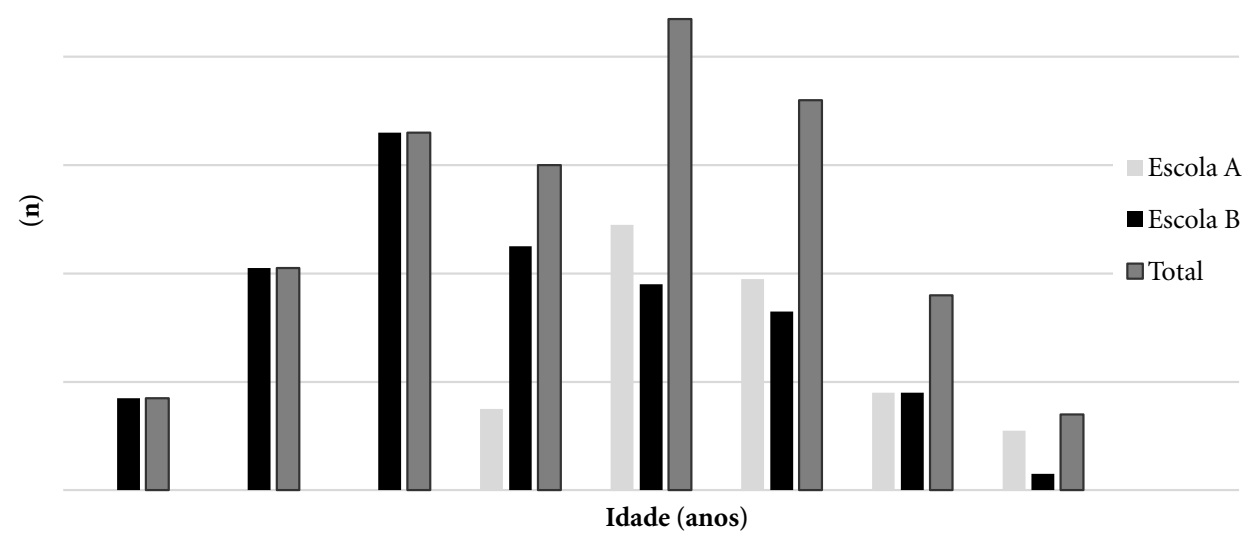

Gráfico 1. Distribuição de alunos de acordo com a faixa etária, 2019.

Fonte: Elaborado pelos autores.

escolas possuem 393 alunos, com 190 do sexo feminino e 203 do sexo masculino.

Conforme mostra o Gráfico 1, foi realizado ponto de coorte segundo as faixas etárias do PNAE. Na escola A foram encontrados 15 alunos na faixa etária entre 11-15 anos, 106 alunos na faixa etária de 16-18 anos e 11 alunos na faixa etária entre 19-30 anos. Já na escola B, 169 alunos encontravam-se na faixa 11-15 anos (64,7\%), sendo 84 do sexo feminino e 85 do sexo masculino. 89 alunos faziam parte da faixa etária de 16-18 anos (34\%) e apenas três alunos pertenciam à faixa etária compreendida entre 19-30 anos (1,3\%).

A Tabela 1 mostra a quantidade de macro e micronutrientes estudados por dia, bem como no final da tabela, a média semanal das preparações em relação aos nutrientes estudados, em que dia um (D1) corresponde às refeições da segunda-feira, das semanas um e três, D2 à terça-feira e assim sucessivamente. Os dias seis a dez (D6 a D10), correspondem às refeições oferecidas de segunda a sexta-feira das semanas dois e quatro, quando as refeições se repetem.

$\mathrm{Na}$ Tabela 2 estão dispostas as recomendações para as faixas etárias 11-15 anos (1500 kcal), 1618 anos $(1700 \mathrm{kcal})$ e $19-30$ anos $(1.600 \mathrm{kcal})$ e seus respectivos percentuais de adequação conforme a idade. Nota-se que, tomando-se como base a média semanal dos macro e micronutrientes das refeições oferecidas encontradas na Tabela 1 , é possível observar que as quantidades encontradas estão abaixo das preconizadas em todas as faixas etárias para quase todos os nutrientes, muitas vezes não alcançado as recomendações da menor faixa etária mínima estudada.

A média semanal de calorias descrita na Tabela 1 foi de 1294,37 kcal. Comparando-se as três faixas etárias estudadas que representam o corpo de alunos das escolas A e B, foi possível observar que as médias encontradas não foram capazes de suprir sequer a necessidade menor faixa etária atendida (11-15 anos) de $1500 \mathrm{kcal}$, atingindo o percentual de adequação de $86,2 \%$ das necessidades energéticas recomendadas pelo Programa. Apenas D5 e D9 alcançaram valores próximos aos de referência para esta menor faixa etária estudada. Vale salientar que, à medida que a faixa etária avança, as necessidades energéticas também aumentam, podendo agravar as situações carenciais e se tornar um grave problema de saúde pública.

Em relação às proteínas, observou-se que em quatro dias (dias 2, 8, 9 e 10) foram alcançados os requerimentos para todas as faixas etárias estudadas, com valores acima de 52 gramas (recomendação máxima da última faixa etária estudada) conforme preconizado pelo PNAE no alcance de $70 \%$ das necessidades/dia para todas as idades, chegando a alcançar 113,4\% de adequação na ultima faixa etária estudada (19-30 anos). Já os piores resultados foram do dia 7 , alcançando baixos índices de adequação em relação às proteínas em todas as faixas etárias, e do dia 6 , com apenas 28,8 gramas por dia, muito abaixo daquele preconizado pelo PNAE.

Assim, com base na média semanal de proteína encontrada, de 46,94 gramas, variou-se ade- 


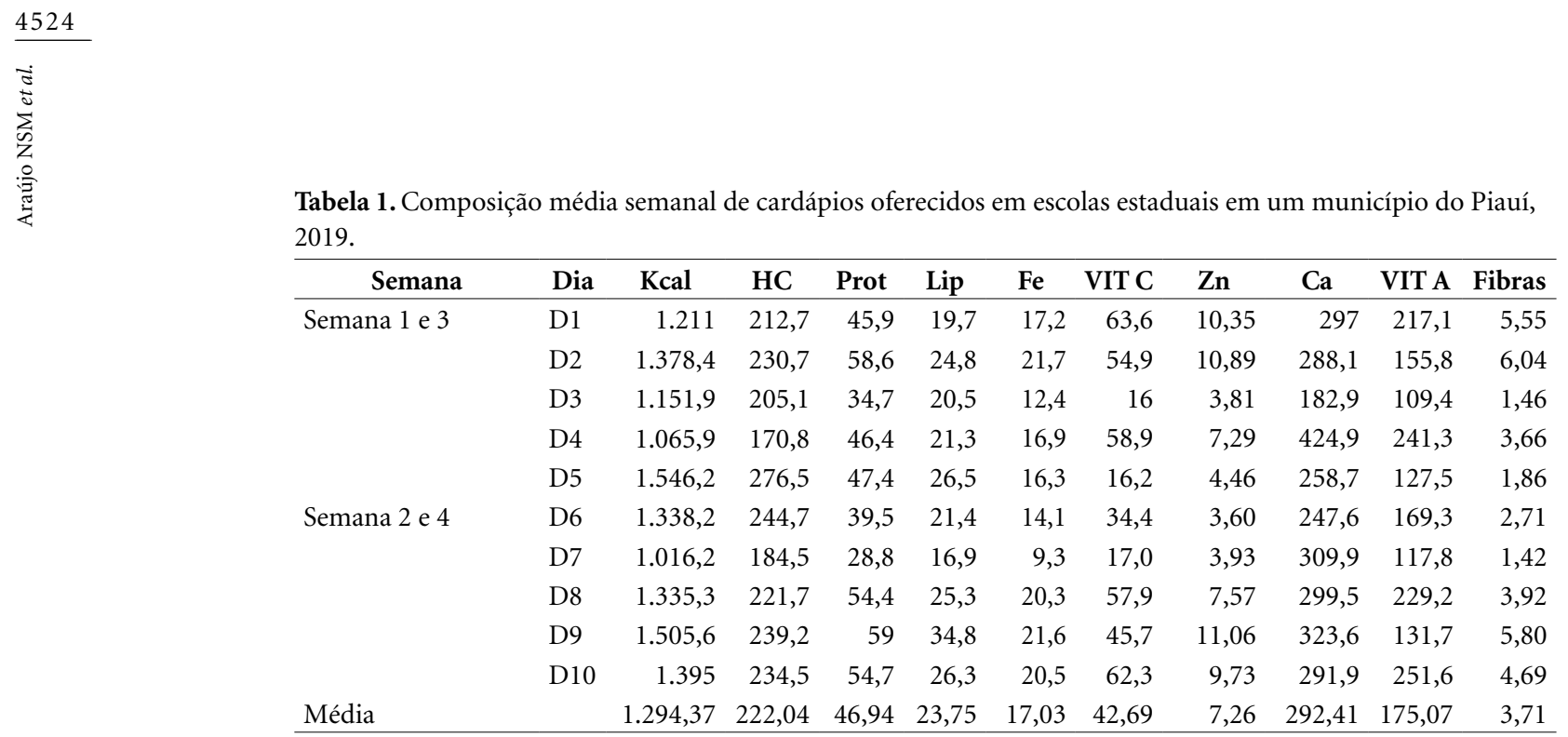

Fonte: Elaborado pelos autores.

Tabela 2. Necessidades de macro e micronutrientes preconizadas pelo PNAE segundo faixa etária x \% adequação, 2019.

\begin{tabular}{lccrrrrrrrrr}
\hline \multicolumn{1}{c}{ Idade } & $\begin{array}{c}\text { Recomendação } \\
\text { (Brasil,2009) }\end{array}$ & Kcal & HC & Prot & Lip & Ferro & VIT C & Zinco & Ca & VIT A & Fibras \\
\hline $11-15$ & Recomendação & 1500 & 243,8 & 46,9 & 37,5 & 7,5 & 42 & 6,3 & 910 & 490 & 21,1 \\
anos & Adequação(\%) & 86,2 & 91 & 99 & 63,3 & 227 & 101 & 115,2 & 32,13 & 35,72 & 17,58 \\
$16-18$ & Recomendação & 1.700 & 276,3 & 50 & 42,5 & 9,1 & 49 & 7 & 910 & 560 & 22,4 \\
anos & Adequação(\%) & 76,1 & 80,36 & 93,8 & 55,8 & 187,1 & 87,1 & 103,7 & 32,13 & 31,26 & 16,56 \\
$19-30$ & Recomendação & 1600 & 260 & 52 & 40 & 9,1 & 61 & 6,8 & 700 & 560 & 22,1 \\
anos & Adequação (\%) & 80,8 & 85,4 & 90,26 & 59,3 & 250,4 & 70 & 106,7 & 41,77 & 31,26 & 16,78 \\
\hline
\end{tabular}

Fonte: Elaborada pelos autores.

quação de $100 \%$ na faixa etária de 11-15 anos, 93,8\% na faixa etária de 16-18 anos e 90,26\% na faixa etária de 19-30 anos. Vale salientar que a média semanal teve um efeito mais positivo, pensando-se em efeitos a longo prazo, do que a avaliação dos valores de proteínas encontrados nas refeições oferecidas diariamente.

Com relação aos carboidratos, observou-se que o valor médio semanal ofertado no cardápio, $222 \mathrm{kcal}$, foi insuficiente para os requerimentos propostos, não atingindo sequer o recomendado pelo PNAE para a faixa etária de 11-15 anos. Na faixa etária 11-15 anos foi encontrado $91 \%$ de adequação, na idade compreendida entre 16-18 anos encontramos $80,36 \%$ de adequação e entre 19-30 anos com adequação em torno de 85\%.

Para os lipídios, os valores estiveram abaixo dos recomendados para todos os grupos etários, em todos os dias de refeições servidas. A média semanal encontrada alcançou para as faixas etárias 11-15 anos o valor de $63,3 \%$ de adequação (referência: 37,5 gramas), 55,8\% adequação para idade entre 16-18 anos (referência: 42,5 gramas) e 59,3\% de adequação na faixa etária entre 19-30 anos (referência: 40 gramas).

A vitamina $C$ atingiu o valor recomendado apenas na faixa etária de 11-15 anos. O melhor resultado encontrado para o cálcio foi no $\mathrm{D} 4$, em que alcançou apenas um valor de 60,5\% de adequação para a faixa etária de 19-30 anos.

Em relação à média semanal para vitamina $\mathrm{A}$ das preparações, foi encontrado o valor de valor de 175,07 miligramas, o que infere em baixos níveis de adequação para as faixas etárias estudadas, como por exemplo, para a faixa etária de 11-15 anos há uma recomendação de 490 miligramas, em que se alcançou apenas $44,3 \%$ de adequação.

Os valores de zinco foram adequados em seis dias de ofertas alimentares. A quantidade de zinco ofertada nas refeições analisadas não foi suficiente para as faixas etárias de 11-15 e 16-18 anos em apenas quatro dias (valores $<5$ miligramas) conforme mostra a Tabela 1. Comparando-se com a média semanal (7,31 miligramas), devido 
alguns dias terem sido encontrados valores bem acima do preconizados pelo PNAE, foi obtida uma média que cobriu os déficit em todas as faixas etárias estudadas, alcançando adequação de $115 \%$ para a faixa etária de 11-15 anos, 103,7\% para a faixa etária de 6-18 anos e 106,7\% para idade entre 19-30 anos.

A quantidade de fibras foi insuficiente para todas as faixas etárias estudadas. A média encontrada foi de 3,71 gramas, valor muito abaixo do recomendado. A fibra foi a variável que apresentou os piores resultados de adequação nutricional, pois alcançou no máximo $52,4 \%$ de adequação no dia 9, para a faixa etária entre 11-15 anos, conforme mostra a Tabela 3.

\section{Discussão}

Os resultados obtidos no presente estudo permitiu observar um déficit/inadequação na elaboração e composição de macro e micronutrientes de cardápios oferecidos a adolescentes que frequentam escolas de tempo integral no município de Picos/PI, onde a maioria dos dias de refeições ofertadas nas escolas não atendeu às necessidades preconizadas pelo PNAE.

O consumo alimentar adequado é condição essencial para o alcance das exigências nutricionais e deve estar de acordo com as necessidades de cada fase da vida, principalmente ao longo da infância e adolescência por serem estágios biologicamente mais suscetíveis ${ }^{21}$.

Em estudo semelhante desenvolvido no município de Americana no estado de São Paulo, a dieta dos escolares revelou-se, em média, deficiente em energia e as refeições consumidas no âmbito dos Centros Integrados de Educação Pú- blica (CIEPs) não alcançaram 70\% das necessidades nutricionais/dia dos alunos preconizada pela legislação ${ }^{22}$. Escolas de tempo integral encontraram adequação em relação à calorias unicamente para a faixa etária de 6-10 anos. Em contrapartida, para os alunos de 11-15 e 16-18 anos foi observada adequação de apenas $73 \%$ e $64 \%$, respectivamente, em relação às recomendações do PNAE para as necessidades diárias, resultado semelhante ao atual estudo ${ }^{23}$.

A análise da composição dos cardápios de escolas de tempo integral realizadas por Dane$\operatorname{lon}^{7}$ revelou que estes se mostraram concordantes com as recomendações estabelecidas no tocante ao conteúdo de proteínas e insuficientes no que diz respeito à expressiva parcela das vitaminas e minerais.

Pesquisadores $^{23}$ encontraram resultados semelhantes para os valores de vitamina $\mathrm{C}$, revelando-se muito abaixo dos valores estabelecidos pelo PNAE, principalmente nas faixas etárias de 16-18 anos e 19-30 anos. Pode-se inferir a este resultado a baixa ingesta e oferecimento de frutas no mapa de cardápio oferecido aos adolescentes, podendo além do prejuízo dos seus efeitos carenciais próprios, comprometer a absorção do nutriente ferro e levá-los a um quadro anemia ferropriva, uma vez que a vitamina $\mathrm{C}$ é um fator promotor de sua absorção.

O cálcio foi um dos nutrientes que alcançou os piores valores ofertados nas preparações. Em nenhum dos dias ofertados foi alcançado o valor de referência sequer para menor faixa etária (1115 anos). Nota-se que o cardápio oferece baixa ingesta de leite e derivados.

Os valores de zinco alcançaram os $70 \%$ das necessidades nutricionais diárias em seis dias de ofertas alimentares nas escolas de tempo integral

Tabela 3. Quantidade de fibras oferecida no mapa de cardápios, Escola A e B, 2019.

\begin{tabular}{|c|c|c|c|c|}
\hline \multirow{2}{*}{ Dias } & \multirow{2}{*}{$\begin{array}{l}\text { Quantidade } \\
\text { ofertada (g) }\end{array}$} & \multicolumn{3}{|c|}{ \% Adequação } \\
\hline & & 11-15 anos & 16-18 anos & 19-30 anos \\
\hline D1 & 10,35 & 49 & 46,2 & 46,8 \\
\hline D2 & 10,89 & 51,6 & 48,6 & 49,2 \\
\hline D3 & 3,81 & 18 & 17 & 17,2 \\
\hline D4 & 7,29 & 34,5 & 32,5 & 33 \\
\hline D5 & 4,46 & 21,1 & 19,9 & 20,18 \\
\hline D6 & 3,6 & 17 & 16,07 & 16,28 \\
\hline D7 & 3,93 & 18,7 & 17,54 & 17,78 \\
\hline D8 & 7,57 & 35,8 & 33,79 & 34,2 \\
\hline D9 & 11,06 & 52,4 & 49,37 & 50 \\
\hline D10 & 9,73 & 46 & 43,4 & 44 \\
\hline
\end{tabular}

Fonte: Elaborada pelos autores. 
no município de Picos/PI. Os resultados corroboram com o estudo ${ }^{24}$ em que foram encontrados valores acima dos recomendados para alunos do $1^{\circ}$ ao $5^{\circ}$ anos. Entretanto, no atual estudo, em alguns dias foram encontrados valores bem abaixo para essa menor faixa etária estudada. Ressalta-se que entre os efeitos da deficiência de zinco se destaca a redução da função imune e o prejuízo para o crescimento durante a infância e a adolescência ${ }^{25}$.

A avaliação do consumo alimentar mostrou adequação no consumo diário de carboidratos, proteínas, lipídios e ferro em estudo desenvolvido em Alegre/ES ${ }^{26}$ o que corrobora com os resultados encontrados no presente estudo, sendo semelhante apenas para adequação do nutriente ferro, que foi alcançada em todas as faixas etárias. Encontrou-se também resultado semelhante na inadequação no consumo de zinco e vitamina A.

No presente estudo, a adequação para vitamina $\mathrm{A}$ piorou à medida que aumentou-se a faixa etária atendida, em que na idade entre 16-18 anos e 19-30 anos, a recomendação para ambas do Programa é de 560 miligramas e alcançou-se uma adequação de apenas $38,8 \%$.

Foi possível observar nos cardápios a baixa oferta de frutas e verduras.

A Resolução CD/FNDE nº 6, de 8 de maio de 2020, publicou novas regras para oferta de frutas e verduras, em que os cardápios planejados para o período parcial deverão ofertar, no mínimo, 280 gramas/estudante/semana, sendo frutas in natura pelo menos dois dias por semana e legumes e verduras pelo menos três dias por semana. Já para os estudantes em período integral, deve-se oferecer, no mínimo, 520 gramas/estudante/semana, sendo frutas in natura pelo menos quatro dias por semana e legumes e verduras pelo menos cinco dias por semana ${ }^{27}$. Destarte, essa nova Portaria tende a assegurar a melhora nos valores de fibras e de alguns micronutrientes para os alunos.

Segundo Freitas ${ }^{28}$, por "saudável”, entende-se um tipo de alimentação que garante nutrientes necessários aos processos fisiológicos para o desenvolvimento físico e mental do indivíduo, em correspondência com os hábitos alimentares regionais.
Além disso, é importante salientar que deve haver um trabalho de porcionamento na distribuição das refeições, visando um maior atendimento em quantidade e qualidade de macro e micronutrientes nas refeiçoes servidas, tensionando melhorar o valor nutricional da alimentação ofertada.

Entretanto, os resultados encontrados demonstram a essencialidade de maior planejamento na elaboração dos cardápios, aspirando atender as necessidades nutricionais dos adolescentes que frequentam escolas de tempo integral, no sentido de haver um melhor rendimento escolar, bem como um bom desenvolvimento fisiológico e biológico desses alunos.

\section{Conclusão}

Em relação às recomendações nutricionais de energia, de macro e micronutrientes preconizadas pelo PNAE para alunos que frequentam escolas de tempo integral no município de Picos/ PI, observou-se que há uma grande inadequação de nutrientes que podem prejudicar o desenvolvimento físico e cognitivo dos adolescentes.

Evidenciou-se, ainda, a necessidade de diferenciação no per capita ofertado para os alunos, bem como o aumento no investimento financeiro por parte dos gestores, visando diminuir o risco nutricional dos alunos, bem como melhor atender às recomendações do PNAE.

Entretanto, é possível concluir que, a alimentação oferecida nas escolas avaliadas não estava adequada para a maioria dos requerimentos nutricionais estabelecidos pelo PNAE e reforça-se a importância no planejamento da elaboração dos cardápios com vistas a assegurar a boa execução do Programa e evitar doenças carenciais de ordem de saúde pública, bem como analisar e discutir os desafios e as potencialidades da alimentação escolar no âmbito global da Segurança Alimentar e Nutricional e surgimento de novas políticas públicas. 


\section{Colaboradores}

NSM Araújo trabalhou na concepção, pesquisa, metodologia e texto final. MFR Antunes, KMC Rolim, SMML Verde e SCM Araújo trabalharam na revisão crítica do artigo. CAB Silva trabalhou na orientação da pesquisa, metodologia e texto final.

\section{Agradecimentos}

Agradecemos à toda equipe da gestão das escolas de tempo integral do município de Picos/PI ligadas à Secretaria Estadual de Educação e Cultura do Estado do Piauí e às nutricionistas responsáveis pela elaboração dos cardápios oferecidos às escolas por colaborar com o presente estudo.

\section{Referências}

1. Brasil. Programa Nacional de Alimentação Escolar. Resolução/CD/FNDE no 26, de 17 de junho de 2013. Dispõe sobre o atendimento da alimentação escolar aos alunos da educação básica no âmbito do Programa Nacional de Alimentação Escolar - PNAE. [documento na Internet]. Ministério da Educação. [acessado 2019 mar 21]. Disponível em: https://www.fnde. gov.br/acesso-a-informacao/institucional/legislacao/ item $/ 4620$-resolu\%C3\%A7\%C3\%A3o-cd-fnde-n $\%$ C2\%BA-26,-de-17-de-junho-de-2013

2. Cesar JT, Valentim EA, Almeida CCB, Schieferdecker MEM, Schmidt ST. Alimentação Escolar no Brasil e Estados Unidos: uma revisão integrativa. Cien Saude Colet 2018; 23(3): 991-1007.

3. Sousa CB, Sampaio GC, Nogueira TR, Alves EAS, Oliveira VA, Santos MM. Study of energy intake and macronutrients of schoolchildren from Teresina $\mathrm{Mu}-$ nicipal Education Networks. Res Soc Develop 2020; 9(6):83963440.

4. Del Ciampo LA, Del Ciampo IRL. A importância do zinco para a saúde do adolescente. Adolescência e Saude 2014; 11(2):80-86.

5. Julián-Almárcegui C, Gómez-Cabello A, González-Agüero A, Olmedillas H, Gómez-Bruton A, Matute-Llorente A, Casajús JA, Vicente-Rodríguez G. The nutritional status in adolescent Spanish cyclists. Nutr Hosp 2013; 28(4):1184-1189.

6. Brasil. Fundo Nacional de Desenvolvimento da Educação. Manual de Educação Integral para obtenção de apoio financeiro através do Programa Dinheiro Direto Na Escola-PDDE, no exercício de 2009. [documento na Internet]. 2009. Ministério da Educação. [acessado 2019 mar 22]. Disponível em: http://www.fnde.gov. br/web/pdde/manual_educacao_integral_2009_retificado.pdf

7. Danelon MAS. Programa de alimentação escolar em unidades de tempo integral: experiências e desafios de gestão [tese]. São Paulo: Universidade de São Paulo; 2007.

8. Mahan LK, Escott-Stump S, Raymond JL. Krause: alimentos, nutrição e dietoterapia. $13^{\mathrm{a}}$ ed. Rio de Janeiro: Elsevier; 2013.

9. Guimarães COS. Estado nutricional antropométrico e composição corporal de crianças e adolescentes com doença falciforme: deficiências de micronutrientes, estado nutricional antropométrico e composição corporal de crianças e adolescentes com doença falciforme em Feira de Santana-BA [dissertação]. Feira de Santana: Universidade Estadual de Feira de Santana; 2018.

10. Cuppari L. Nutrição Clínica no Adulto. Guias de Medicina Ambulatorial e Hospitalar - Nutrição - Nutrição Clínica no Adulto. $4^{\mathrm{a}}$ Edição. Rio de Janeiro: Manole; 2014.

11. Waldvogel-Abramowski S, Waeber G, Gassner C, Buser A, Frey BM, Favrat B, Tissot J. Physiology of iron metabolism. Transfus Med Hemother 2014; 41(3):213221.

12. Garanito MP, Pitta TS, Carneiro JDA. Deficiência de ferro na adolescência. Rev Brasileira de Hematologia e Hemoterapia Hemotherapy 2010; 32 (Supl. 2):45-48.

13. Silva ELG, Mendes MLM, Messias CMB. O consumo alimentar das vitaminas A, c, b9 e zinco por adolescentes de uma escola pública. Revista Baiana de Saúde Pública 2015; 39(3): 598-616. 
14. Mafra D, Cozzolino SMF. Importância do zinco na nutrição humana. Rev Nutr 2004; 17(1):79-87.

15. Assumpcão D, Dias MRMG, Barros MBA, Fisberg RM, Barros Filho AA. Ingestão de cálcio por adolescentes: inquérito de saúde de base populacional. Jornal de Pediatria 2016; 92(3):251-259.

16. Brasil. Ministério da Saúde (MS). Secretaria de Atenção à Saúde. Protocolos clínicos e diretrizes terapêuticas, volume 3. [documento na Internet]. Ministério da Saúde, Secretaria de Atenção à Saúde. Brasília: MS; 2015. [acessado 2020 fev 02]. Disponível em: http:// bvsms.saude.gov.br/bvs/publicacoes/protocolos_clinicos_diretrizes_terapeuticas_v3.pdf

17. Vitolo MR, Campagnolo PDB, Gama CM. Fatores associados ao risco de consumo insuficiente de fibra alimentar entre adolescentes. Jornal de Pediatria 2007; 83(1):47-52.

18. Azevedo L, Martino HSD, Carvalho FG, Rezende M. Estimativa da ingestão de ferro e vitamina $\mathrm{C}$ em adolescentes no ciclo menstrual. Cien Saude Colet 2010; 15(Supl. 1):1359-1367.

19. Brasil. Fundo Nacional do Desenvolvimento da Educação. Conselho Deliberativo. Resolução $n^{\circ} 38$ de 16 de julho de 2009. Dispõe sobre o atendimento da Alimentação Escolar aos alunos da educação básica no Programa de Alimentação Escolar - PNAE. [documento na Internet]. Ministério da Educação. [acessado 2019 mar 21]. Disponível em: https://www.planalto.gov.br/ Consea/static/documentos/outros/fnde.pdf.pdf

20. Pinto SL, Lucena ALN, Da Silva MB, Cardoso LRC, Silva KC. Avaliação da adequação nutricional da alimentação escolar ofertada em uma escola de tempo integral do município de Palmas, Tocantins, Brasil. Revista Cereus 2017; 9(2):92-107.

21. Marques CCA. Consumo de micronutrientes por adolescentes de escolas públicas e privadas [dissertação]. Teresina: Universidade Federal do Piauí; 2019.

22. Silva MV. Estado nutricional de escolares matriculados em Centros Integrados de Educaçao Pública-CIEP'S [tese]. São Paulo: Universidade de São Paulo; 1996.

23. Lucena ALN, Bezerra MS, Cardoso LRC, Silva KC, Pinto SL. Avaliação da adequação nutricional da alimentação escolar ofertada em uma escola de tempo integral do município de palmas, Tocantins, Brasil. Rev Cereus 2017; 9(2):92-107.

24. Bartolazze LA, Cazal MM. Avaliação da composição nutricional e aceitabilidade da alimentação escolar. Revista Ciência em Extensão 2019; 15(3):7-17.
25. Toral N, Rhein SO, Cintra IP, Fisberg M. O papel do zinco na infância e adolescência. Pediatria Moderna 2005; 41(4):158-168.

26. Vaz-Tostes MG, Cardoso-Pires PC, De Paula AH, De Barros AA, Freitas FV, Pinheiro-Sant'Ana HM, Pedrosa RG, Costa NMB. Estado nutricional relativo ao ferro, zinco e vitamina A de pré-escolares inseridos em um programa de educação alimentar e nutricional. HU Revista 2015; 41(3 e 4):163-170.

27. Brasil. Fundo Nacional de Desenvolvimento da Educação. Nota técnica, No 1879810/2020/COSAN/ CGPAE/DIRAE, 2020. Alterações dos aspectos de Alimentação e Nutrição e de Segurança Alimentar e Nutricional da Resolução CD/FNDE nº 6, de 8 de maio de 2020. [documento na Internet]. Gabinete da Presidência. [acessado 2020 jul 05]. Disponível em: https://www.fnde.gov.br/index.php/programas/pnae/ pnae-area-gestores/pnae-notas-tecnicas-pareceres -relatorios

28. Freitas MCS, Minayo MCS, Ramos LB, Fontes GV, Santos LA, Souza EC, Santos AC, Mota SE, Paiva JB, Bernardelli TM, Demétrio F, Menezes I. Escola: lugar de estudar e de comer. Cien Saude Colet 2013; 18(4):979-985.

Artigo apresentado em 20/11/2020

Aprovado em 26/05/2021

Versão final apresentada em 28/05/2021

Editores-chefes: Romeu Gomes, Antônio Augusto Moura da Silva 